\title{
Dose Point Kernel calculation and modelling with nuclear medicine dosimetry purposes
}

\author{
Ignacio Scarinci*广 \\ LIIFAMIRX - Laboratorio de Investigación e Instrumentación en Física Aplicada a la Medicina \\ e Imágenes por Rayos X - University of Córdoba; Argentina \\ E-mail: fidel.scarinci@gmail.com
}

\section{Mauro Valente}

Institute of Physics E. Gaviola - CONICET, Córdoba; Argentina

LIIFAMIRX - Laboratorio de Investigación e Instrumentación en Física Aplicada a la Medicina

e Imágenes por Rayos X - University of Córdoba; Argentina

E-mail: valentedfamaf.unc.edu.ar

\section{Pedro Pérez}

Institute of Physics E. Gaviola - CONICET, Córdoba; Argentina

LIIFAMIRX - Laboratorio de Investigación e Instrumentación en Física Aplicada a la Medicina

e Imágenes por Rayos X - University of Córdoba; Argentina

Department of Physics - FCF-QyN - University of Río IV; Argentina

E-mail: pperezlefamaf.unc.edu.ar

Monoclonal labeled antibodies are commonly used for radioimmunotherapy purposes in nuclear medicine The procedure is based on the deposition of specific radiactivity concentrations on tumoral regions with the aim of treating tumoral diseases. Patient metabolism produces nonuniform organ activity distributions, but there are some traditional approaches for internal dosimetry assuming uniform spatial distribution of activity within organ or tissues of interest. When patient-specific dosimetry needs to be performed, these methods may involve non negligible uncertainties. Treatment evaluation and preliminary planning require the estimation of absorbed dose distribution and this goal can be achieved by different approaches. For example, it is possible to perform Monte Carlo simulations for numerical dose assessments; whereas analytical calculations can be carried out by convolution of activity distribution using Dose Point Kernels. Besides, it is often found that some internal dosimetry approaches assume simplified conditions of infinite ad homogeneous media for radiation transport and corresponding dose deposition.

This work presents a method for internal dosimetry in nuclear medicine by means of Dose Point Kernels. The proposed method is capable of performing calculations over heterogeneous systems. The starting point is a set of suitable kernels previously calculated by Monte Carlo simulations in different tissues. Thus, dose distribution is obtained by the proposed hybrid approach involving both numerical and analytical methods. Preliminary tests on simplified geometries demonstrated the capability of the developed technique; whereas computation of patient-specific internal dosimetry suggest promising performance. Hence, the developed method may constitute a valuable tool for nuclear medicine dosimetry purposes.

X Latin American Symposium on Nuclear Physics and Applications (X LASNPA),

1-6 December 2013

Montevideo, Uruguay 


\section{INTRODUCTION}

A well known method for radioimmunotherapy is the administration of monoclonal labeled antibodies performed by depositing specific radiactivity concentrations within specific regions, organs or tissues. Biokinetics characteristics of these antibodies produce non-uniform activity distributions. However, even until today there are still some internal dosimetry approaches assuming uniformity to simplify further calculations for nuclear medicine purposes. As expected, these kind of approaches limitate reliability of dose estimations in nuclear medicine for both diagnostic or therapeutic modalities [1].

It is necessary to assess dose distribution for treatment evaluation and planning when using radionuclides. There are different techniques to this scope based on numerical or analytical methods [2]. Monte Carlo simulations are the most commnoly found techniques for assessing dose distribution numerically. On the other hand, analytical methodologies can be performed by convolution of activity distribution by means of Dose Point Kernels (DPK) having also the capability for patientspecific calculations that are not attainable by other simplified analytical approaches like $S$-factors [3].

Actually, most of dosimetry assessments in clinics are performed by simplifications of the Boltzmann radiation transport equation. The more common assumptions regard homogeneity and infinite extensions useful for application of linearity principles. Contrary, Monte Carlo simulations have capability for performing full radiation transport thus achieving reliability and accuracy not available by analytical approaches. Nowadays modern technology allows fast and easy data processing along with high performance in computing, so that Monte Carlo simulations appear as a promising tool for the radiation transport study over soft tissues [4] among others useful applications for internal dosimetry in nuclear medicine.

This work is focused on the utilization of the Monte Carlo method for the calculation of DPK in different biological tissues with the aim of constructing a DPK database wide enough to be useful for performing patient-specific dosimetry in nuclear medicine. Thereby, an hybrid modality between analytical and numerical methods was developed for computing absorbed dose distribution for $\beta^{+}, \beta^{-}$and $\gamma$ radionuclides typically used in nuclear medicine practices [5].

\section{THEORETICAL BACKGROUND AND METHODS}

\subsection{Dose Point Kernel}

The concept of Dose Point Kernel arises from the simplified situation of considering a point source embedded in an infinite homogeneous and isotropic medium. It can be obtained in the framework of the continuous slowing down approximation (CSDA) in terms of the stopping power function $S(E)$. Then, the scaled DPK, denoted as $s D P K$, can be described as follows

$$
\operatorname{sDPK}\left(x, E_{0}\right)=4 \pi \rho r^{2} R_{C S D A} \Phi\left(r, E_{0}\right)
$$

\footnotetext{
* Speaker.

${ }^{\dagger}$ www.famaf.unc.edu.ar/ valente
} 
where $x$ represents the dimensionless quantity $r / R_{C S D A}, E_{0}$ is the energy of the corresponding monoenergetic point source, $\rho$ is the mass density, $R_{C S D A}$ is the particle range in the CSDA and $\Phi$ is the specific absorbed fraction given by $S(E(r)) / 4 \pi \rho r^{2} E_{0}$.

Therefore, according to this description, the $S D P K$ quantity can be written in terms of the $S(E)$ as $[4,5]$ :

$$
\operatorname{sDPK}\left(x, E_{0}\right)=\frac{S\left(E\left(x, R_{C S D A}\right)\right)}{\langle S\rangle}
$$

\subsection{Methods}

It was proposed by sifferent authors [4,5] to compute $s D P K$ calculations for $\beta^{-}$emitters by means of suitable tallies consisting on concentric shells within homogeneous media. This information is the starting point for further dose distribution calculations applying linearity principles for superimposing the corresponding contributions. This work presents an adapted Monte Carlo simulation routine capable of perfoming full radiation transport to estimate $s D P K$ for both monoenergetic sources and radionuclides.

Calculation of $S D P K$ requires the evaluation of the $R_{C S D A}$, which have been obtained in this case from databases included in the distribution of the PENELOPE 2008 Monte Carlo main code [6]. Actually, the PENELOPE code, largely tested for medical physics and nuclear medicine specifically [5, 7], includes a dedicated subrutine called "Table" providing values of $R_{C S D A}$ for different media being indistinguishable from the NIST database.

Full radiation transport was carried out using physics packages from the PENELOPE code. Radiation-matter interaction models were extracted from these packages in order to develop specific routines for computation of $s D P K$ in different biological tissues and other material typically involved in medical physics like liquid water. There were constructed the required geometries, asigned the corresponding material properties to each region and finally tallies were defined by a dedicated mesh consisting on concentric shells to compute energy deposition. Therefore, outputs from Monte Carlo calculations consisted on mean energy deposition -and standard deviationswithin concentric spherical shells of thickness $0.025 R_{C S D A}$. There were considered sixty shells in order to perform energy deposition up to $1.5 R_{C S D A}$, which may be large enough to include the deposition of quite the whole energy emitted by the source $[4,5]$. In the case of radionuclides, it was considered the "effective" $R_{C S D A}$, which was defined as the $R_{C S D A}$ corresponding to the mean energy of emission spectrum $\bar{E}$ in the medium been investigated. Following typical preliminary tests for simulation convergence [5], it was demonstrated that acceptable uncertainties (lower than $0.1 \%$ ) were attained for both monoenergetic and radionuclides sources running $10^{7}$ primary particles.

\subsection{Hybrid model for internal dosimetry}

As mentioned above, the method proposes the utilization of convolution techniques for assessing absorbed dose distribution once $s D P K$ are already calculated for corresponding medium and source.

In this framework the absorbed dose $D(\vec{r})$ at any location $\vec{r}$ can be obtained by means of the convolution kernel $\mathbb{K}_{D}(\vec{r})$ and the cumulated activity $\mathbb{A}_{\text {cum }}(\vec{r})$ tensors as follows: 


$$
D(r)=\mathbb{K}_{D}(\vec{r}) * \mathbb{A}_{\text {cum }}(\vec{r})
$$

At this point, it was proposed to apply Fourier transforms to solve the convolution operation. Considering a discrete space defined by the 3D voxel level representations, it becomes possible to assess absorbed dose in any voxel, denoted by indices $(i, j, k)$, according to:

$$
D(i, j, k)=\mathbb{F}^{-1}\left\{\mathbb{F}\left\{\mathscr{K}_{D}(i, j, k)\right\} \cdot \mathbb{F}\left\{\mathscr{A}_{\text {cum }}(i, j, k)\right\}\right\}
$$

where $\mathbb{F}^{-1}$ and $\mathbb{F}$ are the discrete operators for Fourier transforms representing the inverse and forward Fourier transforms, respectively. Additionally, $\mathscr{K}_{D}$ and $\mathscr{A}_{\text {cum }}$ are the $3 \mathrm{D}$ voxel level representation of kernel and cumulated activity, respectively.

Solutions for the last equation can be obtained by means of numerical algorithms like Fast Fourier Transform (FFT) routine provided by many software languages for several toolkits.

\section{RESULTS}

Figure 1 reports some of the results typically obtained from the computation of $s D P K$ for both monoenergetic and radionuclides sources. These results are compared against those published by Botta et al using the FLUKA Monte Carlo simulation code for radiation transport and dose delivery.

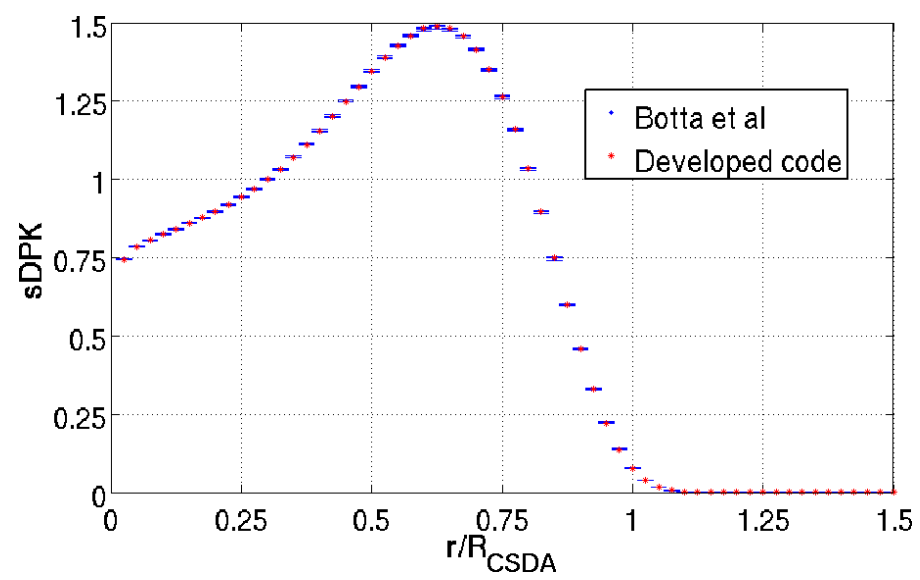

Figure 1: In-water $s D P K$ for $1 \mathrm{MeV} \beta^{-}$source compared against Botta et al

As described in previous section, $s D P K$ were calculated for many biological tissues and other materials like water. Figure 2 shows $s D P K$ for a selected group of tissues corresponding to a definite kinetic energy.

It was decided to include only three materials in graphic just to provide better visualization because they are representative of the general behavior replicated in all cases. As usually, liquid water was used as reference while other materials and biological tissues were compared in reference to water. There are reported $s D P K$ for compact bone and lungs because they are those with properties somewhat relative different to water, particularly mass density. However, it has to be remarked that all studied materials and tissues (dry air, brain, cortical bone, skeletal muscle, striated 


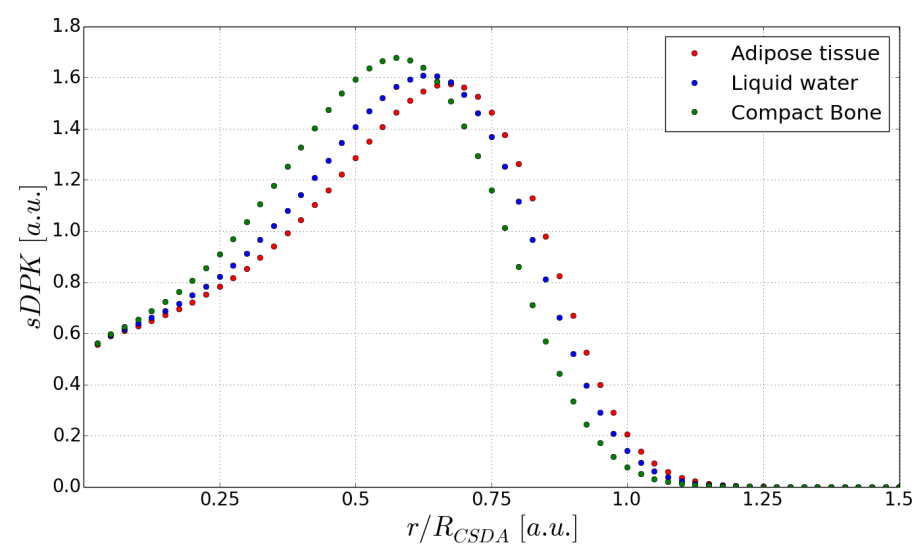

Figure 2: $s D P K$ for $100 \mathrm{keV} \beta^{-}$source in different media

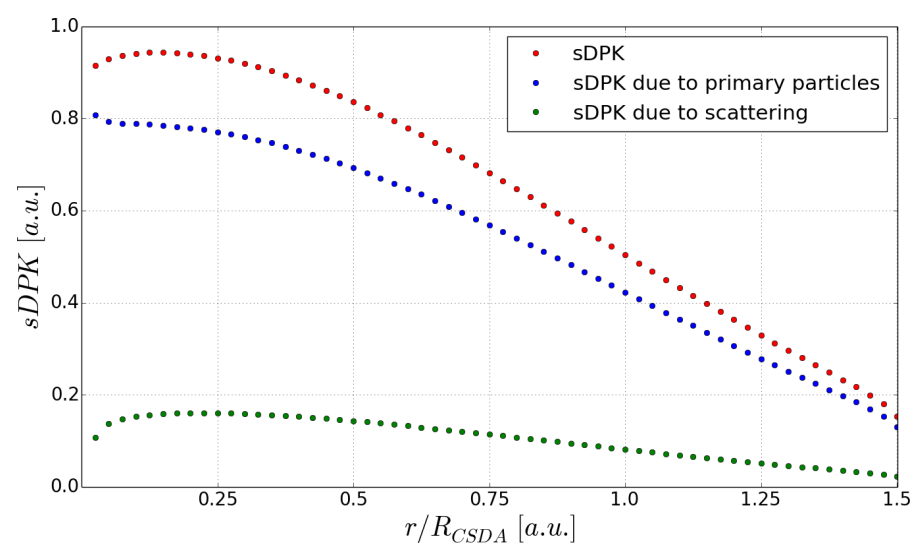

Figure 3: $s D P K$ for $1 \mathrm{MeV} \beta^{-}$source in liquid water

muscle, skin, blood, adipose tissue, soft tissue, among others) showed similar trend and in all cases the corresponding $s D P K$ lyes between the extremes determined by compact bone and lung tissue, as expected.

In addition, it was developed a dedicated module for calculating simultaneously and separately dose contributions due to primary or scattering radiation. As example of the obtained results, Figures 3 and 4 present the $s D P K$ where primary and scattering components were discriminated for both monoenergetic $(1 \mathrm{MeV})$ and radionuclide $\left({ }^{90} \mathrm{Y}\right)$ sources, respectively.

Finally, there were perfomed direct applications to routinary clinical cases. It was selected a typical SPECT-CT dual scan with the aim of performing patient-specific dosimetry as daily required in nuclear medicine services. Figure 5 reports the an image fusion on a slice between patient anatomy from CT and metabolism from SPECT.

Absorbed dose distribution was calculated to this patient-specific case by means of $s D P K$ convolution according to the proposed method and the obtained results are showed in Figure 6.

In this example it was selected to perform the calculation for ${ }^{90} Y$ radionuclide, but would be exactly the same procedure for any other radiopharmaceutical once its $s D P K$ were already known. 


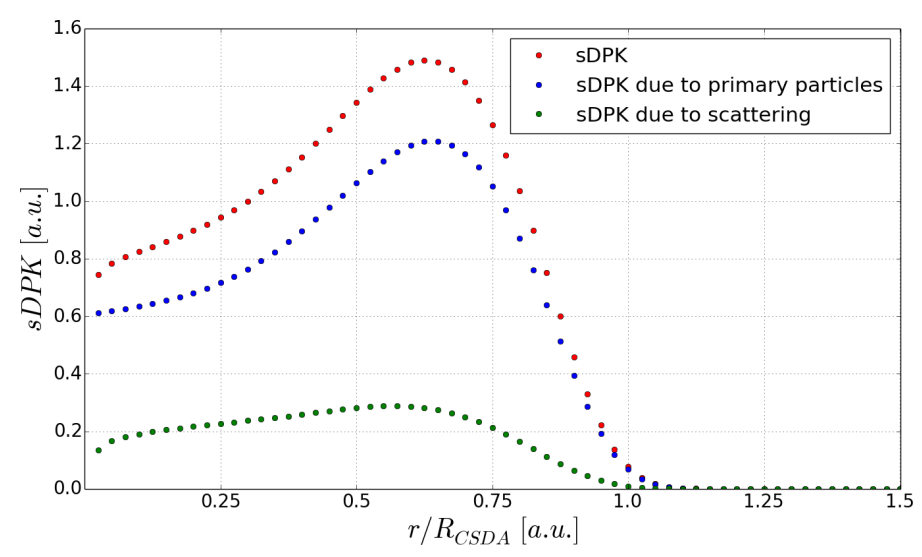

Figure 4: $s D P K$ for ${ }^{90} Y$ source in dry air

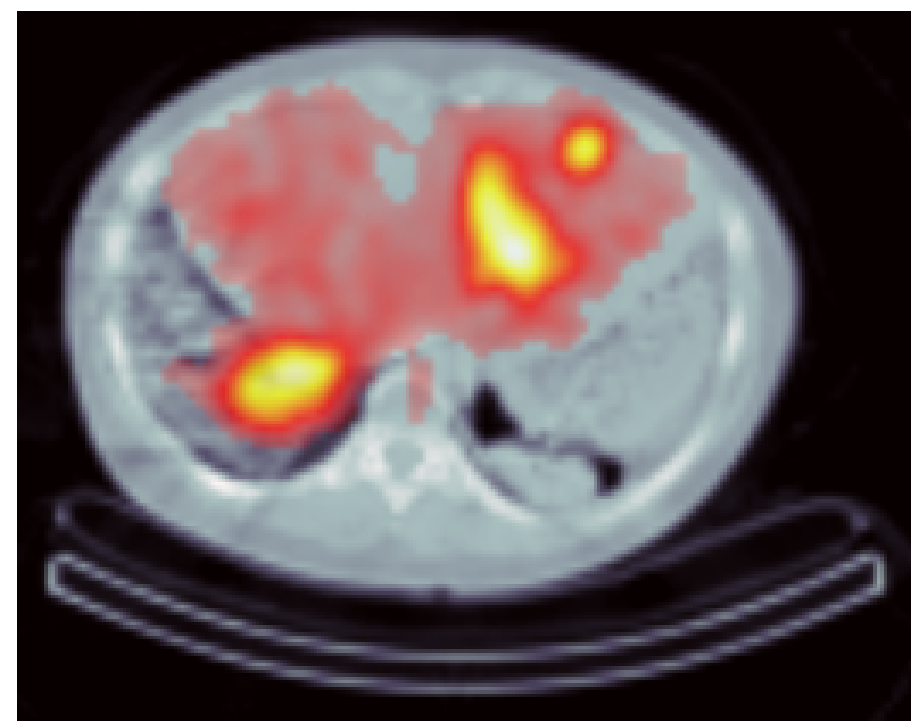

Figure 5: Fusion of anatomy and activity

\section{CONCLUSIONS AND DISCUSSIONS}

As a first comment it should be pointed out that the proposed method based on hybrid approach for internal dosimetry assessment has shown remarkable feasibility. There were successfully computed absorbed dose distributions within complex geometries by means of kernel convolution involving $S D P K$ values previously calculated. In this sense, it was also possible to develop a dedicate Monte Carlo routine for the computation of $s D P K$ in different biological tissues and materials typically used in medical physics. According to the reported results for determination of $s D P K$ in different biological materials, it has to be highlighted that the obtained results are in agreement with validated data published by other authors [4]. Thus, considering this preliminary benchmark as a possitive suggestion it might be possible to expect that the developed code is capable of reliable $s D P K$ calculations. Moreover, it was shown that the developed simulation code was actually capable of quantifying the different contributions to absorbed dose. This issue may be useful or actually necessary to perform accurate radiobiological dosimetry, because of the differences in radiobiolog- 


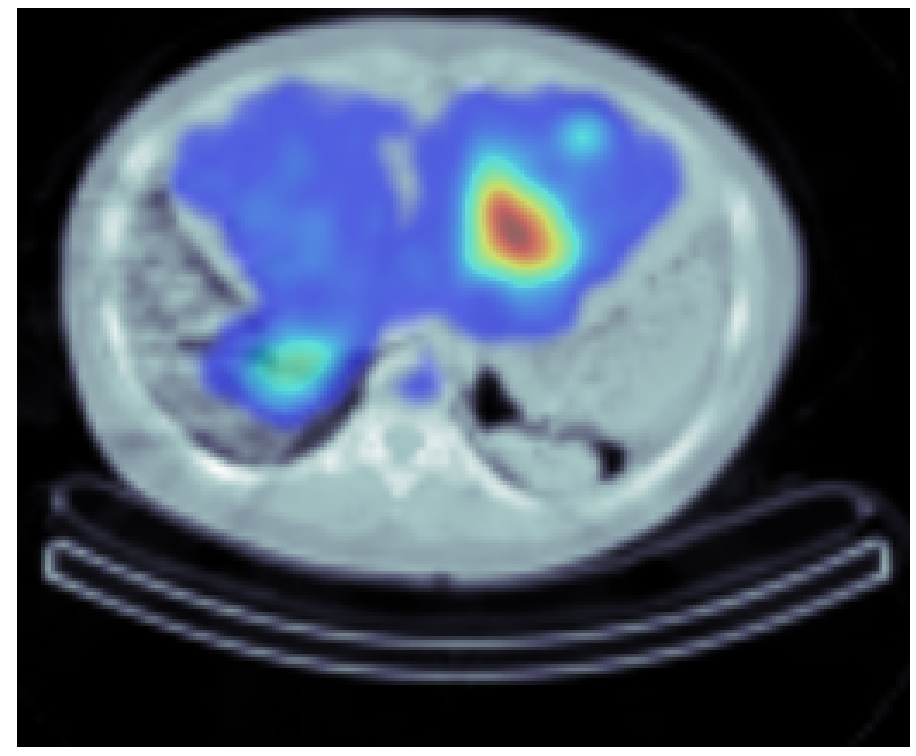

Figure 6: Dose distribution using the hybrid $s D P K$ convolution method

ical effectiveness of particles with different linear energy transfer. In addition, the discrimination of the different contributions to the total $s D P K$ constitutes a valuable tool for improving analytical models that might be focused in incorporating scattering effects.

Finally, it has to be underlined the satisfactory performance for radiation transport in complex geometries along with the capability of defining source distributions directly from dicom formated files for further construction of voxelized mesh and position for primary particle (desintegration). This feature is the main core of the system for performing patient-specific internal dosimetry. The obtained results, like Figure 6, showed promising performance that needs to be widely and exhaustively benchmarked against other methods before considering to proceed with clinical applications.

\section{References}

[1] G. Sgouros, Dosimetry of Internal Emitters, J Nucl Med, 2005; 46(1):18S-27S.

[2] W.E. Bolch et al, MIRD Pamphlet No. 17: The Dosimetry of Nonuniform Activity Distributions-Radionuclide S Values at the Voxel Level, J Nucl Med, 1999; 40:11S-36S.

[3] W.V. Prestwich et al, Beta Dose Point Kernels for Radionuclides of Potential Use in Radioimmunotherapy, J Nucl Med, 1989; 30:1036-1046.

[4] F. Botta et al, Calculation of electron and isotopes dose point kernels with FLUKA Monte Carlo code for dosimetry in nuclear medicine therapy, Med Phys, 2011; 38(7):3944-3954.

[5] P. Pérez et al. Dosimetry for Beta-Emitter Radionuclides by Means of Monte Carlo Simulations. 12 Chapter on Nuclear Medicine. Intech. 2010.

[6] F. Salvat, J. Fernández-Varea, J. Sempau. PENELOPE, A code system for Monte Carlo simulation of electron and photon transport. 2008 version, NEA France.

[7] H. Uusijärvi, N. Chouin N, P. Bernhardt, L. Ferrer, M. Bardiés, E. Forssell-Aronsson. Comparison of Electron Dose-Point Kernels in Water generated by the Monte Carlo Codes, PENELOPE, GEANT4, MCNPX, and ETRAN. Cancer Biother. Radiopharm. 24(4):461-467, 2009. 